\title{
Nanoscale Domain Stability in Organic Monolayers on Metals
}

Z. Suo ${ }^{*}$ and Y.F. Gao

Mechanical and Aerospace Engineering Department and Princeton Materials Institute

Princeton University, Princeton, NJ 08544

\section{G. Scoles}

Chemistry Department and Princeton Materials Institute

Princeton University, Princeton, NJ 08544

\begin{abstract}
Certain organic molecules, such as alkanethiols, can adsorb on metals to form monolayers. Sometimes domains appear in the monolayers. For example, an incomplete monolayer may form islands, and a mixed-composition monolayer may separate into distinct phases. During annealing, molecules diffuse on the metal surface. The domain boundary energy drives the domains to coarsen. The contact potential between dissimilar domains drives the domains to refine. On the basis of existing experimental information, we suggest that the competition between coarsening and refining should stabilize certain domain patterns. We formulate a free energy functional to include the effects of mixed species, domain boundary, and contact potential. An approximate energy minimization estimates the equilibrium domain size. We derive a diffusion equation consistent with the free energy functional. The numerical solution of the diffusion equation follows the evolution of the monolayers from a random initial concentration field to patterns of dots and stripes. The patterns do not have any long-range order unless some form of anisotropy is introduced. We also discuss the practical implications of the theory and, in particular, the possibility of guided self-assembly.
\end{abstract}

*Author to whom correspondence should be addressed: email, suo@princeton.edu; tel., 609-258-0250; fax, 609-258-5877. 


\section{Introduction}

An alkanethiol molecule, $\mathrm{HS}\left(\mathrm{CH}_{2}\right)_{\mathrm{n}} \mathrm{X}$, consists of a thiol group $\mathrm{HS}$ at one end, an alkyl chain $\left(\mathrm{CH}_{2}\right)_{\mathrm{n}}$ in the middle, and a tail group $\mathrm{X}$ at the other end. As illustrated in Fig. 1, when a clean gold substrate is in contact with an alkanethiol solution, the molecules adsorb on the gold surface to form a self-assembled monolayer (SAM). ${ }^{1-3}$ The thiol groups bond to the gold surface, the alkyl chains attract one another through the van der Waals force, and the tail groups are exposed at the surface. Alkanethiol monolayers have been used as a model system to study many phenomena. For instance, SAMs can control surface properties (e.g., adhesion and wetting). ${ }^{4}$ Patterned SAMs (e.g., by microcontact printing) are used to fabricate devices, and to confine cells and biomolecules in desired regions on a substrate. ${ }^{5-7}$

Under certain conditions, a SAM spontaneously forms domains. For example, for an incomplete monolayer, patches of the monolayer coexist with patches of the bare metal. ${ }^{8-10}$ (The "bare metal" can actually be covered by the lying-down phase. ${ }^{1}$ ) Also, when a monolayer of dissimilar alkanethiols fully covers the metal surface, different phases may coexist. ${ }^{9-12}$ Unless the distinction is important, we will refer to the patches as domains, be they standing-up phase, lying-down phase, or bare metal. The domains observed so far have sizes ranging from nanometers to micrometers, and do not form any regular pattern. Is the domain size set by thermodynamic equilibrium, or by kinetics? Can the domains be stimulated to form some regular patterns, such as an array of stripes or a lattice of dots? Within the alkanethiol family, properties vary considerably with the alkyl chain length and the tail group, giving rise to a large parameter space which can be used to tailor experiments. Regular domain patterns of a controllable size would open new applications of theses systems. Consequently, it is significant to consider these questions theoretically, even though definitive experiments are lacking. A 
predictive theory will point to fruitful experiments with alkanethiols on gold, as well as with other molecule-substrate systems.

This study was also prompted by the observation of equilibrium domain pattern formation in monolayers of several types. Examples include atomic monolayers on solid surfaces, ${ }^{13-19}$ and molecular monolayer at the air-water interface (i.e. Langmuir films). ${ }^{2,20-23}$ The adsorbed atoms or molecules are mobile. Domains coarsen to reduce the total length of the domain boundaries. The residual stresses, or the presence of electric dipoles in a monolayer, induce an elastic or electrostatic field, so that the domains may refine to reduce the free energy associated with the field. It is the competition between the domain boundary energy and the field energy that leads to the equilibrium domain patterns. ${ }^{23-30}$ The observed domain sizes range from nanometers to hundreds of micrometers.

The object of this paper is to examine, in principle, if regular domain patterns can form in alkanethiol monolayers on gold. Section II discusses phase separation, domain coarsening, and domain refining, drawing on existing experimental data that relate to alkanethiols on gold. The theory follows closely those developed for Langmuir films. We will look at two types of models. In the first type, one assigns a constant dipole moment density to each domain, allows a step jump in the dipole moment density across a domain boundary, and represents the domain boundary by a curve with a line tension. ${ }^{23-27}$ In the second type, one represents a multi-domain monolayer by a continuum concentration field, describes a domain boundary by a gradient in the concentration field, and prescribes the free energy as a functional of the concentration field. ${ }^{28,29}$ The two models have the same physical content, but different mathematical representations. We will adopt the first model in Section II to discuss concepts, and adopt the second in the subsequent sections to simulate the emergence and evolution of complex domain patterns. 
The two systems - Langmuir films and SAMs — have similar thermodynamics. Section III formulates a free energy functional to describe the effects of mixed species, domain boundaries, contact potential, and electrostatic field. In Section IV, we minimize the free energy by assuming sinusoidal concentration fields, arriving at an estimate of the equilibrium domain size. Because the long-range interaction is mediated through the electrostatic field in the space above the monolayer, one can alter this interaction by introducing dielectrics or metals into the space. We will discuss the possibility to change domain patterns this way. The effect of surface stress is also discussed, using available experimental data.

During annealing, the rate processes in the two systems are different. Domains in a Langmuir film change by viscous flow in the monolayer and water, although molecules may also diffuse on the surface. ${ }^{21,31,32}$ Domains in a SAM on metal change by molecular diffusion alone. Electronic transport in the metal is much faster than molecular diffusion on the surface. Consequently, as molecules diffuse on the surface, electrons flow in the metal rapidly, the electric potential in the metal equalizes, and the electrostatic field in the space above the monolayer adjusts accordingly. In Section V, we derive a diffusion equation compatible with the free energy functional, and numerically simulate the annealing process. The simulation shows that long-range ordering of the patterns is extremely slow, unless symmetry is suitably broken.

\section{Phase separation, domain coarsening, and domain refining}

When a gold substrate is in contact with an alkanethiol solution, two kinds of mass transport, i.e. adsorption-desorption and diffusion, proceed simultaneously. First consider the case that the solution contains a single species of alkanethiol. Initially molecules adsorbed on the surface form islands. As more molecules adsorb, the islands connect, and the remaining bare 
gold surface appears as monolayer-deep vacancy islands. Further adsorption causes the vacancy islands to shrink and disappear. Finally a monolayer completely covers the gold surface. If surface diffusion is negligible during adsorption, the islands (if they form at all) in an incomplete monolayer clearly cannot reach any equilibrium pattern. Consequently, a necessary condition to form an equilibrium domain pattern is to break the contact between the solution and the metal before the monolayer completes. In subsequent annealing, molecules diffuse on the surface to change the domain patterns.

Similar considerations apply to the case that the solution contains two alkanethiol species, A and B. As pointed out by Folkers et al., ${ }^{32}$ if gold is kept in contact with the solution, allowing the molecules on gold to exchange with those in the solution, in equilibrium the monolayer will have a single phase. Again, a necessary condition to stabilize two phases in a monolayer is to break the contact between the solution and the metal at some point. The monolayer covers the entire surface, and the amount of the two components in the monolayer has a suitable ratio. For the two phases to equilibrate in annealing, the enthalpy of mixing has to overcome the entropy of mixing. Let $C$ be the concentration of the monolayer, namely, the fraction of surface sites on gold occupied by B-alkanethiols. During annealing, the molecules can diffuse on the surface, but the amount of either species remains constant, so that the average concentration of the monolayer, $C_{0}$, remains invariant. Figure 2 illustrates the free energy of mixing $g(C)$ for a homogeneous monolayer. The two wells at $C_{\alpha}$ and $C_{\beta}$ correspond to the two phases. When $C_{\alpha}<C_{0}<C_{\beta}$, the monolayer separates into the two phases in equilibrium.

In the above discussion, we have neglected the effect of contact potential and surface stress in stabilizing domains when the monolayer is in contact with the solution, or when the free 
energy of mixing has a single well. The phenomena may occur in a small concentration or temperature range, and have been discussed in Refs. 27 and 28.

Alkanethiol molecules form strong bonds to gold surface, and are not very mobile at room temperature. The diffusivity of alkanethiols on gold is estimated to be $D=10^{-21} \mathrm{~m}^{2} / \mathrm{s}$. ${ }^{33}$ For the concentration field to change over a length scale $L$, the time needed scales as $t \sim L^{2} / D$. For example, if the domain size is $10 \mathrm{~nm}$, the time scale is $\sim 10^{5} \mathrm{~s}$. It has been observed that nanoscale islands in an incomplete monolayer under atmospheric conditions coarsen at room temperature in days. ${ }^{8}$ To accelerate pattern formation, the monolayer can be annealed above room temperature, as long as the molecules do not evaporate appreciably, and the phases are still stable.

The excess free energy of the domain boundaries, i.e., the line tension, drives coarsening. For domains to be stable, a refining action must exist to prevent domains from growing too large. We now examine how the contact potential drives domains to refine. Regard the monolayer and a few top layers of gold atoms as an interfacial system. Across the thickness of this system, the positive and the negative electric charges are unevenly distributed, resulting in an electric dipole normal to the surface. Now consider two gold substrates, one covered with a monolayer of phase $\alpha$, and the other with a monolayer of phase $\beta$. When the two substrates are connected, electrons flow from one substrate to the other, until the chemical potentials of electrons in the two substrates are equilibrated. Due to the difference in the two monolayers, the electric potential in space near $\alpha$ differs from that near $\beta$, say $\phi_{\alpha}<\phi_{\beta}$. The difference, $U=\phi_{\beta}-\phi_{\alpha}$, is known as the contact potential, and can be measured by the Kelvin method. ${ }^{34-36}$ The contact potential sets up an electrostatic field in the space, and a charge density field on the metal surface. $^{37}$ 
Figure 3 illustrates a monolayer composed of two kinds of domains, $\alpha$ and $\beta$. The period $\lambda$ represents the domain size. The metal occupies the lower half space $x_{3}<0$, and the monolayer coincides with the $\left(x_{1}, x_{2}\right)$ plane. The space above the monolayer is occupied by air. The electrostatic energy stored in the space depends on the magnitude of the contact potential, but not on how it is set up. Imagine that the contact potential can be varied from zero to its final magnitude $U$. This can be accomplished, for example, by starting with a pure $\alpha$-phase monolayer, and gradually converting some patches into the $\beta$-phase. When the contact potential is zero, the charge on the metal surface is zero. As the contact potential increases, electrons flow in the metal, setting up a net positive charge $+Q$ on the metal surface under the $\beta$ domains, and a net negative charge $-Q$ under the $\alpha$ domains. Because the electrostatic field equations in the space are all linear, $Q$ is linear in $U$ (Fig. 4a). The slope of the line is the inverse of the capacitance of the system. When the contact potential changes from 0 to $U$, the external agent that effects the change does the work $\frac{1}{2} Q U$. This work is stored as the electrostatic energy in the space above the monolayer. It is always a positive quantity.

In reality, the contact potential $U$ is held constant by the molecular difference between the two domains. The contact potential acts like a battery, and the $\beta$ and $\alpha$ domains act like two electrodes. The metal substrate serves as a wire connecting the electrodes. When transporting an amount of charge $Q$ from the $\alpha$ domains to the $\beta$ domains, the constant voltage $U$ does work $Q U$. Having done this work, the "battery" reduces the free energy. Consequently, the electric free energy is the electrostatic energy in space minus the work done by the constant contact potential, namely, $\frac{1}{2} Q U-Q U=-\frac{1}{2} Q U$. The electric free energy is always a negative quantity. At a constant voltage, the higher the capacitance, the larger the charge, and the lower the free 
energy. That is, to reduce the electric free energy, the system evolves toward a configuration of high capacitance.

We can now understand the refining action due to the contact potential. In the discussion above, we kept the domain pattern fixed. Now allow the domain pattern to change by molecular diffusion. In this process, $U$ is constant, but both the charge $Q$ on the metal surface and the electrostatic field in space change. As the domain size decreases from $\lambda_{2}$ to $\lambda_{1}$, the capacitance increases (Fig. 4b). The trend is analogous to a parallel-electrode capacitor (Fig. 4c). The charge $Q$ increases as the domain size decreases. The electric free energy is reduced if the domain size decreases, so that the contact potential drives the domains to refine.

The above understanding has an important practical consequence. If a dielectric or a metal is introduced in the space above the monolayer, the capacitance of the system changes and so do the equilibrium domain patterns. This provides a means for guided self-assembly. The significance of this will be discussed in Section V.

For the parallel-electrode capacitor (Fig. 4c), the free energy variation with the separation gives rise to the familiar attractive force between the two electrodes, which must be balanced by a pair of some other forces pulling the electrodes apart. In a poly-domain monolayer, the free energy variation with the domain size gives rise to a driving force for molecular diffusion. We will derive an explicit expression for this driving force in Section V.

\section{Free energy as a functional of the concentration field}

In this section, we represent a poly-domain monolayer by a continuum concentration field, $C\left(x_{1}, x_{2}\right)$, and a domain boundary by a gradient in the concentration field. First consider a surface of gold covered with a homogenous monolayer of concentration $C$. Denote $\phi$ as the 
contact potential between this surface and a reference surface, say, a gold surface covered with a monolayer of pure A. We assume that the contact potential is linear in the concentration:

$$
\phi=\zeta C .
$$

That is, the dipole moment of an individual molecule, either A or B, is assumed to be unaffected by the presence of other molecules on the substrate. The metal is covered by pure A at $C=0$, and by pure B at $C=1$ Consequently, the slope $\zeta$ in (1) equals the contact potential between a substrate covered by pure B and another substrate covered by pure A. This contact potential can be measured by connecting these two substrates in the Kelvin method.

Figure 5 illustrates the interface between the air and the metal. We assume that the thickness of the interfacial system is small compared to the domain size, and is negligible in calculating the electrostatic field. Denote the electric potential in the space by $\Psi\left(x_{1}, x_{2}, x_{3}\right)$. The electric potential in the bulk of the metal is constant, taken to be zero. In the space, at a point immediately above the monolayer, $x_{3}=0^{+}$, the electric potential equals the contact potential:

$$
\Psi\left(x_{1}, x_{2}, 0\right)=\phi\left(x_{1}, x_{2}\right)=\zeta C\left(x_{1}, x_{2}\right) .
$$

Let the electric charge per unit area on the metal surface be $\sigma\left(x_{1}, x_{2}\right)$. Applying Gauss' law to a small volume containing an element of the interfacial system, one confirms that the electric displacement component in the space immediately above the monolayer is $D_{3}\left(x_{1}, x_{2}, 0\right)=\sigma\left(x_{1}, x_{2}\right)$. Consequently, the surface charge density relates to the electric potential in space as

$$
\sigma\left(x_{1}, x_{2}\right)=-\varepsilon_{0} \frac{\partial \Psi}{\partial x_{3}}, \quad x_{3}=0
$$

The field equations are standard. The electric field relates to the gradient of the electric potential as $\mathbf{E}=-\nabla \Psi$. The electric displacement is linear in the electric field, namely, $\mathbf{D}=\varepsilon_{0} \mathbf{E}$. 
We assume that the upper half space is free of charges, so that the electric displacement is divergence-free, $\nabla \cdot \mathbf{D}=0$. Consequently, the electric potential in the air satisfies the Laplace equation:

$$
\nabla^{2} \Psi=0
$$

The electrostatic energy stored in the space is the same as the work done in building up the contact potential from zero to $\phi$, namely,

$$
\int \frac{1}{2} \mathbf{E} \cdot \mathbf{D} d V=\int \frac{1}{2} \phi \sigma d A
$$

The integral on the left-hand side extends over the volume of the half space above the surface, and the integral on the right-hand side extends over the area of the surface. Equation (5) can also be confirmed by using the field equations and the divergence theorem. We assume that the system as a whole is neutral, $\int \sigma d A=0$. Equation (5) shows that the electrostatic energy in the space above the monolayer vanishes when the contact potential is uniform over the surface, as expected.

Next we examine the free energy of the interfacial system. The interfacial energy density, $\Gamma$, takes an unusual from. Assume that $\Gamma$ is a function of the concentration $C$, the concentration gradient $\nabla C$, and the surface charge density $\sigma$. Expanding the function into the Taylor series to the leading order terms in $\nabla C$ and $\sigma$, we have

$$
\Gamma=g+h \mid \nabla C^{2}-\phi \sigma
$$

where $g, h$ and $\phi$ are in general functions of $C$. The term $g(C)$ is the surface energy density when the concentration is uniform and the surface charge vanishes. Indeed, the function $g(C)$ is the free energy of mixing (Fig. 2), taken to be an input of the model. Because the interfacial energy density is independent of the direction of the concentration gradient, the leading term in 
$\nabla C$ is quadratic. Following Cahn and Hilliard ${ }^{38}$, we interpret this term as a continuum representation of the domain boundary energy, with $h$ being a positive constant. In the expansion (6), we keep the third term linear in $\sigma$, but neglect terms which are higher order in $\sigma$. As discussed in Section II, when $\phi$ is held a constant for the interfacial element, the work done by $\phi$ to add charge $\sigma$ to the element is $\sigma \phi$, which reduces the free energy.

The excess free energy of the interfacial object is the integral of $\Gamma$ over the area of the surface. The free energy $G$ of the system — the SAM on metal and the space above-is the sum of the electrostatic energy stored in the space, Eq. (5), and the excess free energy of the interfacial object, namely,

$$
G=\int\left(g+h \mid \nabla C^{2}-\frac{1}{2} \phi \sigma\right) d A
$$

In summary, this section defines a thermodynamic system by prescribing a procedure to calculate its free energy. The free energy is a functional of the concentration field. Given a concentration field $C\left(x_{1}, x_{2}\right)$, one determines the electric potential $\Psi\left(x_{1}, x_{2}, x_{3}\right)$ by solving the Laplace equation (4) subject to the boundary condition (2), and then calculates the surface charge density $\sigma\left(x_{1}, x_{2}\right)$ according to (3). Equation (7) gives the free energy of the system. The concentration field evolves to minimize this free energy. This paper will assume that the space above the monolayer is occupied by air. If a dielectric or a metal is present in the space during annealing, the effect can be included in the boundary value problem.

\section{An approximate analysis of equilibrium domain sizes}

An equilibrium domain pattern corresponds to a concentration field that minimizes the free energy functional (7). By definition $C$ varies between 0 and 1 . During annealing, the metal is no 
longer in contact with the alkanethiol solution, so that the average concentration of the monolayer, $C_{0}$, is fixed. To estimate the equilibrium domain size, we minimize the free energy over a particular family of concentration fields:

$$
C\left(x_{1}, x_{2}\right)=C_{0}+C_{1} \sin \left(\frac{2 \pi x_{1}}{\lambda}\right) .
$$

This family represents an array of periodic stripes oriented along the $x_{2}$ direction. The average concentration is indeed $C_{0}$. The amplitude of fluctuation, $C_{1}$, and the period, $\lambda$, are varied to minimize the free energy.

A combination of (1) and (8) gives the contact potential

$$
\phi=\zeta C_{1} \sin \left(\frac{2 \pi x_{1}}{\lambda}\right) .
$$

Matching this boundary condition, the solution to the Laplace equation gives the electric potential in the upper half space:

$$
\Psi=\zeta C_{1} \sin \left(\frac{2 \pi x_{1}}{\lambda}\right) \exp \left(-\frac{2 \pi x_{3}}{\lambda}\right) .
$$

The electric potential decays exponentially as $x_{3} \rightarrow \infty$, the decay length being $\lambda / 2 \pi$. The surface charge is calculated according to (3), giving

$$
\sigma=\varepsilon_{0} \zeta C_{1}\left(\frac{2 \pi}{\lambda}\right) \sin \left(\frac{2 \pi x_{1}}{\lambda}\right) .
$$

The surface charge is in phase with the contact potential (9), as expected.

The average energy per unit area is the integral (7) over one period, divided by the period. The calculation gives

$$
\bar{G}=\bar{g}+\frac{C_{1}^{2}}{4}\left[2 h\left(\frac{2 \pi}{\lambda}\right)^{2}-\varepsilon_{0} \zeta^{2}\left(\frac{2 \pi}{\lambda}\right)\right] .
$$


The average energy of mixing, $\bar{g}$, is independent of the wavelength. The two terms in the bracket result from, respectively, the domain boundary and electrostatics. The trends discussed in Section II can now be seen clearly in (12). The domain boundary energy reduces when the wavelength increases, and drives the domains to coarsen. The electrostatic energy reduces when the wavelength decreases, and drives the domains to refine.

The free energy is quadratic in $(2 \pi / \lambda)$, and reaches its minimum at the wavelength

$$
\lambda_{0}=\frac{8 \pi h}{\varepsilon_{0} \zeta^{2}} .
$$

Using $\varepsilon_{0}=8.85 \times 10^{-12} \mathrm{~F} / \mathrm{m}, h=10^{-21} \mathrm{~J}$ and $\zeta=0.5 \mathrm{~V}$, we find that $\lambda_{0}=11 \mathrm{~nm}$. Before taking this estimate seriously, we should discuss the validity of the analysis, and the field of variability of the parameters.

In reaching (13), we have sought the energy minimizer among candidates of a very small family of concentration fields, Eq. (8). The sinusoidal concentration profile is a reasonable approximation when the domain size is not too large compared to the domain boundary width. This happens when the refining action is strong, or when the coarsening action is weak (e.g., when the binary monolayer is held just below the critical temperature). When the domain size is, say, more than ten times the domain boundary width, however, the concentration profile is close to a square wave, which should be represented by a Fourier series of many wavelengths. An analysis following this line of thought shows that the equilibrium domain size is larger than that given by (13). ${ }^{42}$ In the limit that the domain size is much larger than the domain boundary width, the line tension model is appropriate, giving another estimate of the equilibrium domain size: ${ }^{25,26}$

$$
\lambda_{\infty}=2 \pi a \exp \left(\frac{2 \pi \gamma}{\varepsilon_{0} U^{2}}+1\right)
$$


where $a$ is a cutoff radius (close to molecular dimensions, $a \sim 1 \mathrm{~nm}$ ), $\gamma$ the line tension, and $U$ the contact potential between the two domains. The step jump of the contact potential at the domain boundary makes the electrostatic energy unbounded, and the cutoff radius is introduced to regularize the problem. Taking $\gamma=10^{-12} \mathrm{~N}$ and $U=0.5 \mathrm{~V}$, we find that $\lambda_{\infty}=292 \mathrm{~nm}$.

Next we discuss the variability field of the parameters that determine the equilibrium domain size. We have assumed that the space above the monolayer is occupied by air. If a highpermittivity dielectric fluid lies above the monolayer during annealing, the equilibrium domain size will reduce accordingly. Of course, the presence of a dielectric fluid, rather than the air, may modify the contact potential and the domain boundary energy somewhat. Independent measurements of these quantities have to be made. Similarly, if one places a high-permittivity dielectric solid at a small gap above the monolayer during annealing, one can, in principle, even tune the equilibrium domain size by adjusting the gap.

Contact potentials have been measured for alkanethiols on gold. ${ }^{35,36}$ The potential increases linearly with the alkyl chain length by $0.0093 \mathrm{~V}$ per $\mathrm{CH}_{2}$ unit. The potential changes also when the tail group changes; variation between $-0.75 \mathrm{~V}$ to $+0.60 \mathrm{~V}$ has been reported. One can even incorporate polar groups in the middle of the alkyl chain to increase the contact potential without compromising the functionality of the tail group.

We are unaware of any measurement of the domain boundary energy in alkanethiol monolayers. The line tension of the phase boundary in Langmuir films has been measured experimentally, a representative order of magnitude being $\gamma=10^{-12} \mathrm{~N} .{ }^{22}$ Considering the similarity of the inter-molecular forces involved in Langmuir films and in SAMs, we expect that the magnitude of the line tension should be comparable in the two systems. Note that the line tension decreases as the temperature increases, and vanishes above the critical temperature. 
Because the inter-molecular forces are weak, the critical temperature is not too high, and is typically within experimental reach.

Given the large variability in the parameters, one should expect very different equilibrium domain sizes in different systems. That is, the equilibrium domain size should be tunable.

When both the surface stress and contact potential are present, a combination of the above analysis and that in Suo and $\mathrm{Lu}^{28}$ gives the equilibrium domain size:

$$
\lambda_{0}=\frac{8 \pi h}{\varepsilon_{0} \zeta^{2}+\frac{(1-v) \xi^{2}}{\mu}},
$$

where $\mu$ is the shear modulus, $v$ is Poisson's ratio, and $\xi$ is the slope of the surface stress as a function of the concentration. The relative importance of the electrostatic and elastic interaction is quantified by a dimensionless ratio

$$
R=\frac{\mu \varepsilon_{0} \zeta^{2}}{(1-v) \xi^{2}}
$$

The surface stress has been measured for alkanethiols on gold, giving $0.017 \mathrm{~N} / \mathrm{m}$ per $\mathrm{CH}_{2}$ unit. ${ }^{39}$ The available data indicate that the effect of the surface stress and that of the contact potential are comparable for alkanethiols on gold. However, if one introduces high-permittivity dielectrics in the space above the monolayer, and incorporates polar groups into the molecules, the electrostatic interaction can be altered by orders of magnitude.

\section{Diffusion equation and the need to break symmetry}

In this section, we consider the diffusion process, in which the monolayer starts from an arbitrary initial concentration field, and evolves to a stable domain pattern. We derive a diffusion equation, following a standard procedure in nonequilibrium thermodynamics. ${ }^{28,38}$ Imagine a curve on the substrate surface. When some number of A-molecules crosses this curve, 
to maintain the integrity of the monolayer, an equal number of B-molecules must cross the curve in the opposite direction. Denote the unit vector lying in the surface normal to the curve by $\mathbf{m}$. Let $\mathbf{I}$ be a vector field in the surface, such that $\mathbf{I} \cdot \mathbf{m}$ is the number of B-molecules across a unit length of the curve.

When the concentration on an element of the surface varies by $\delta C$, the same number of Bmolecules must move into the element from the neighboring regions on the surface, namely,

$$
\Lambda \delta C=-\nabla \cdot(\delta \mathbf{I})
$$

where $\Lambda$ is the number of surface sites per unit area. Combining (7) and (17), we find the variation of the free energy:

$$
\delta G=\frac{1}{\Lambda} \int(\boldsymbol{d}) \cdot \nabla\left(\frac{\partial g}{\partial C}-2 h \nabla^{2} C-\zeta \sigma\right) d A
$$

In deriving (18), we have used the fact that the term $\phi \sigma$ in (7) is quadratic in $C$. We have also discarded integrals along curves on the surface, assuming periodic boundary conditions.

Define the diffusion driving force $\mathbf{f}$ as the free energy reduction associated with a molecule relocating by a unit distance. Comparing this definition and (18), we obtain an expression for the diffusion driving force:

$$
\mathbf{f}=-\frac{1}{\Lambda} \nabla\left(\frac{\partial g}{\partial C}-2 h \nabla^{2} C-\zeta \sigma\right)
$$

When the diffusion driving force vanishes, the free energy variation vanishes, and the concentration field reaches equilibrium. The quantity in the parenthesis is a chemical potential. A concentration field is in equilibrium when the chemical potential is constant over the surface. Obviously, a homogeneous monolayer is an equilibrium state, which can be unstable. We are interested in stable, inhomogeneous equilibrium states. 
In general, for an arbitrary concentration field, the driving force does not vanish —it drives the diffusion flux. Assume that the diffusion flux, $\mathbf{J}$, is linearly proportional to the driving force, namely, $\mathbf{J}=M \mathbf{f}$, where $M$ is the mobility of the molecules on the surface. The conservation of molecules requires that $\Lambda \partial C / \partial t=-\nabla \cdot \mathbf{J}$. These considerations lead to the diffusion equation

$$
\frac{\partial C}{\partial t}=\frac{M}{\Lambda^{2}} \nabla^{2}\left(\frac{\partial g}{\partial C}-2 h \nabla^{2} C-\zeta \sigma\right) .
$$

To evolve the concentration field numerically, we need to specify the free energy of mixing $g(C)$. Any function with two wells, as shown in Fig. 2, will serve the purpose. To be specific, we assume that the binary monolayer is a regular solution, with the free energy of mixing given by

$$
g(C)=\Lambda k_{B} T[C \log C+(1-C) \log (1-C)]+\Lambda \omega C(1-C)
$$

where $k_{B}$ is Boltzmann's constant, and $T$ the absolute temperature. The parameter $\omega$ measures the magnitude of the enthalpy of mixing. When $\omega / k_{B} T<2$, the entropy of mixing prevails, and $g(C)$ has a single well. When $\omega / k_{B} T>2$, the enthalpy of mixing prevails, and $g(C)$ has two wells.

A comparison of the first two terms in (20) leads to a length:

$$
b=\left(\frac{h}{\Lambda k_{B} T}\right)^{1 / 2} .
$$

This length scales the distance over which the concentration changes from the level of one phase to that of the other. From (20) and (21), we note that the diffusivity scales as $D \sim M k_{B} T / \Lambda$. To resolve events occurring over the length scale $b$, the time scale is $b^{2} / D$. This consideration defines a time scale

$$
\tau=\frac{h}{M\left(k_{B} T\right)^{2}}
$$


To evolve the concentration field according to (20), at each time step, for a given concentration field, we need to solve the electrostatic boundary value problem, and calculate the surface charge field. This can be done by an area integral of a Green's function. The integral is singular, and extends over the entire surface. This approach would take a great deal of computation time. Rather, we will solve the electrostatic boundary value problem in the Fourier space. Consider the Fourier transform

$$
C\left(x_{1}, x_{2}, t\right)=\int_{-\infty-\infty}^{+\infty+\infty} \hat{C}\left(k_{1}, k_{2}, t\right) \exp \left(i k_{1} x_{1}+i k_{2} x_{2}\right) d k_{1} d k_{2}
$$

To ensure that $C\left(x_{1}, x_{2}, t\right)$ is real-valued, the two Fourier components $\hat{C}\left(k_{1}, k_{2}, t\right)$ and $\hat{C}\left(-k_{1},-k_{2}, t\right)$ must be complex conjugate. Because the electrostatic field is governed by linear equations, we only need to determine the electrostatic field for an individual Fourier component, and then superimpose all the components. For a pair of components, $\hat{C}\left(k_{1}, k_{2}, t\right)$ and $\hat{C}\left(-k_{1},-k_{2}, t\right)$, the concentration field is

$$
C=2 \operatorname{Re}\left[\hat{C}\left(k_{1}, k_{2}, t\right) \exp \left(i k_{1} x_{1}+i k_{2} x_{2}\right)\right]
$$

where Re stands for the real part of a complex number. The contact potential is

$$
\phi=2 \zeta \operatorname{Re}\left[\hat{C} \exp \left(i k_{1} x_{1}+i k_{2} x_{2}\right)\right]
$$

This prescribes the boundary condition at $x_{3}=0$ for the electrostatic field in the upper half space. One can readily confirm that the solution to the Laplace equation is

$$
\Psi=2 \zeta \operatorname{Re}\left[\hat{C} \exp \left(i k_{1} x_{1}+i k_{2} x_{2}-k x_{3}\right)\right]
$$

where $k=\sqrt{k_{1}^{2}+k_{2}^{2}}$. This electric potential matches the boundary condition (26), and vanishes as $x_{3} \rightarrow+\infty$. The surface charge density is

$$
\sigma=2 \varepsilon_{0} k \zeta \operatorname{Re}\left[\hat{C} \exp \left(i k_{1} x_{1}+i k_{2} x_{2}\right)\right]
$$


Normalizing the time by $\tau$ and the spatial coordinates by $b$ in (20), and taking the Fourier transform on both sides, we obtain that

$$
\frac{\partial \hat{C}}{\partial t}=-k^{2} \hat{P}-2 k^{4} \hat{C}+\rho k^{3} \hat{C}
$$

where $\rho=8 \pi b / \lambda_{0}$ is a dimensionless parameter, and $\hat{P}\left(k_{1}, k_{2}\right)$ is a Fourier component of the function

$$
P(C)=\log \left(\frac{C}{1-C}\right)+\frac{\omega}{k_{B} T}(1-2 C),
$$

which comes from the derivative of the free energy of mixing (21).

Remarkably, Eq. (29) is identical to that of a monolayer on an isotropic elastic substrate, where the surface stress drives domain refining. ${ }^{29}$ We will the use the same numerical method to evolve the concentration field. The function $P(C)$ is nonlinear in $C$, so that $\hat{P}$ at a given point in the $\left(k_{1}, k_{2}\right)$ plane depends on $\hat{C}$ at all points in the $\left(k_{1}, k_{2}\right)$ plane. Consequently, (29) evolves $\hat{C}$ at all points in the $\left(k_{1}, k_{2}\right)$ planes simultaneously, in a coupled manner. Numerical simulation proceeds as follows. Start with a known concentration field $C\left(x_{1}, x_{2}, t_{0}\right)$ at time $t_{0}$. Calculate $P$ according to (30), and $\hat{P}$ by using the Fast Fourier Transform (FFT). Also obtain $\hat{C}$ by FFT. Equation (29) updates the field $\hat{C}$ for a small time step. Take an inverse FFT to obtain the updated $C$ field. Repeat the procedure for many time steps to evolve the concentration field over a long period of time. More details of numerical implementation can be found in Refs. 29 and 40.

In numerical simulations, we take $\omega / k_{B} T=2.2$, so that $g(C)$ has two wells at $C_{\alpha}=0.249$ and $C_{\beta}=0.751$. We take $\rho=2$, so that $\lambda_{0}=4 \pi b$, and the equilibrium domain size is about one order of magnitude larger than the domain wall width. We restrict the calculation within a 
$256 b \times 256 b$ square cell. Periodic boundary conditions are used to replicate the cell to the entire monolayer. Figure 6 shows two simulation results taken from $\mathrm{Lu}$ and Suo, ${ }^{29}$ which was originally intended for patterns stabilized by surface stress. Now if we interpret $\lambda_{0}$ by (15), the same simulation describes pattern evolution under combined actions of surface stress and contact potential.

Figure 6a shows the concentration field after the annealing time $t=10^{5} \tau$. The initial concentration field randomly fluctuates around the average value $C_{0}=0.5$. At around $t=10^{2} \tau$, the concentration field has already separated into two phases of meandering stripes. The pattern and the feature size hardly change between $10^{2} \tau$ to $10^{5} \tau$.

Figure $6 \mathrm{~b}$ shows a pattern at time $t=4 \times 10^{6} \tau$, initiated from a concentration field randomly fluctuated around the average value $C_{0}=0.4$. The dots are established around $t=10^{2} \tau$. Further annealing does not change the size of the dots appreciably, but improves the spatial ordering of the dots. At $t=4 \times 10^{6} \tau$ shown in Fig. $7 \mathrm{~b}$, the pattern consists of grains, each grain being a triangular lattice consisting of fewer than ten dots across.

Both meandering stripes and disordered dots have been observed in alkanethiol monolayers. ${ }^{8-12}$ Some of these experiments were carried out with the monolayers in contact with the alkanethiol solution at room temperature, so that the adsorption process affected the domain patterns. Alkanethiol molecules diffuse slowly on gold at room temperature, so that the observed domains may not be of the equilibrium size.

Figure 6 also clearly shows the effect of symmetry on pattern formation. The model is isotropic, with no preferred orientation in the plane of the monolayer. Consequently, stripes of all orientations are equally possible, so are lattices of all orientations. Improving the long-range order by annealing alone takes a long time. A powerful way to form patterns with long-range 
ordering is to break the symmetry. In a series of papers, ${ }^{41-43}$ we have studied the effect of symmetry breaking of various modes on domain patterns, assuming surface stress stabilizes the domains. Domains stabilized by contact potential offer additional opportunities. For example, during annealing, one can place an anisotropic dielectric crystal at a small distance above the monolayer. The presence of the crystal affects the electrostatic field, and can set a preferred orientation to guide the pattern. Similarly, one can make a metal pattern on a solid using lithography, and then place the patterned solid over the monolayer. During annealing, this lithographic pattern can guide the pattern formation in the monolayer. The concept is analogous to the Lithographically-Induced Self-Assembly (LISA). ${ }^{44-46}$ We will report details of these intriguing possibilities in subsequent work.

\section{Concluding remarks}

On the basis of available experimental data and theories, we suggest that alkanethiol SAMs on gold should form domain patterns under certain conditions. Upon annealing, alkanethiol molecules diffuse on the gold surface, and the domain pattern may evolve into an equilibrium state. The domain boundary energy drives the domains to coarsen, and the contact potential drives the domains to refine. The competition sets an equilibrium domain size, which can be varied by varying the alkyl chain length, by incorporating polar groups into the molecules, by placing a high-permittivity dielectric liquid above the monolayer, and by changing the temperature. In an isotropic system, the domain patterns are not organized at long-range. Various modes of symmetry breaking may guide domains into periodic lattices. SAMs with ordered domains of controllable sizes would open new possibilities for applications. Further experiments and theoretical work need to be carried out to ascertain the premises, and to explore 
new opportunities, particularly those of guided self-assembly. The concepts are also applicable to monolayers of other molecules, notably biomolecules adsorbed on solid surfaces. Provided molecules on surfaces are mobile, the combined effects of surface stress and contact potential may be strong enough to stabilize domains of desired sizes.

\section{Acknowledgements}

The work is supported by the Department of Energy through grants DE-FG02-99ER45787 and DE-FG02-93ER45503, and by the National Science Foundation through grant CMS9820713. We gratefully acknowledge helpful discussions with D.J. Srolovitz, K. Vanderlick, and Y. Hu. 


\section{References}

(1) Schreiber, F. Prog. Surf. Sci. 2000, 65, 151-256.

(2) Ulman, A. An Introduction to Ultrathin Organic Fs: from Langmuir-Blodgett to SelfAssembly; Academic Press: Boston,1991.

(3) Dubois, L. H.; Nuzzo, R. G. Ann. Rev. Phys. Chem. 1992, 43,437-463.

(4) Bain, C. D.; Whitesides, G. M. J. Am Chem. Soc. 1989, 111, 7164-7175.

(5) Kumar, A.; Biebuyck, H. A.; Whitesides, G. M. Langmuir 1994, 10, 1498-.

(6) Delamarche, E.; Schmid, H.; Bietsch, A; Larsen, N. B.; Rothuizen, H.; Michel, B.; Biebuyck, H. J. Phys. Chem. B 1998, 102, 3324-.

(7) Schön, J. H.; Meng, H.; Bao, Z. Science 2001, 294, 2138-2140.

(8) Barrena, E.; Ocal, C.; Salmeron, M. J. Chem. Phys. 1999, 111, 9797-9802.

(9) Tamada, K.; Hara, M.; Sasabe, H.; Knoll, W. Langmuir 1997, 13, 1558-1566.

(10) Stranick, S. J.; Parikh, A. N.; Tao, Y.T.; Allara, D. L.; Weiss, P. S. J. Phys. Chem. 1994, 98, 7636-7646.

(11) Stranick, S. J.; Atre, S. V.; Parikh, A. N.; Wood, M. C.; Allara, D. L.; Winograd, N.; Weiss, P. S. Nanotechnology 1996, 7, 438-442.

(12) Hobara, D.; Ota, M.; Imabayashi, S.; Niki. K.; Kakiuchi, T. J. Electroanal. Chem. 1998, 444, 113-119.

(13) Kern, K.; Niehus, H.; Schatz, A.; Zeppenfeld, P.; Goerge, J.; Comsa, G. Phys. Rev. Lett. 1991, 67, 855-858.

(14) Leibsle, F. M.; Flipse, C. F. J.; Robinson, A. W. Phys. Rev. B 1993, 47, 15865-15868.

(15) Parker, T. M.; Wilson, L. K.; Condon, N. G.; Leibsle, F. M. Phys. Rev. B 1997, 56, 64586461. 
(16) Brune, H. M.; Giovannin, K.; Bromann, K.; Kern, K. Nature 1998, 394, 451-453.

(17) Pohl, K.; Bartelt, M. C.; de la Figuera, J.; Bartelt, N. C. J. Hrbek, R. Q. Hwang, Nature 1999, 397, 238-241.

(18) Plass, R.; Last, J. A.; Bartelt, N. C.; Kellogg, G. L. Nature 2001, 412, 875-875.

(19) Ellmer, H.; Repain, V.; Rousset, S.; Croset, B.; Sotto, M.; Zeppenfeld, P. Surf. Sci. 2001, 476, 95-106.

(20) Petty, M. C. Langmuir-Blodgett Films; Cambridge University Press: Cambridge, UK, 1996.

(21) McConnell, H. M. Annu. Rev. Phys. Chem. 1991, 42, 171-195.

(22) Benvegnu, D.J.; McConnell, H. M. J. Phys. Chem. 1992, 96, 6820-6824.

(23) Andelman, D.; Brochard, F.; Joanny, J.-F. J. Chem. Phys. 1987, 86, 3673-3681.

(24) Seul, M.; Andelman, D. Science 1995, 267, 476-483.

(25) Alerhand, O. L.; Vanderbilt, D.; Meade, R. D.; Joannopoulos, J. D. Phys. Rev. Lett. 1988, $61,1973-1976$.

(26) Ng, K.-O.; Vanderbilt, D. Phys. Rev. B 1995, 52, 2177-2183.

(27) Hannon, J.B.; Tersoff, J.; Tromp, R.M. Science 2002, 295, 299-301.

(28) Suo, Z.; Lu, W. J. Mech. Phys. Solids 2000, 48, 211-232.

(29) Lu, W.; Suo, Z. J. Mech. Phys. Solids 2001, 49, 1937-1950.

(30) Mate, C. M.; Kao, C.-T.; Somorjai, G. A. Surf. Sci. 1988, 206, 145-168.

(31) Stone, H. A.; Ajdari, A. J. Fluid Mech. 1998, 369, 151-173.

(32) Folkers, J. P.; Laibinis, P. S.; Whitesides, G. M.; Deutch, J. J. Phys. Chem. 1994, 98, 563571.

(33) Imabayashi, S.; Hobara, D.; Kakiuchi, T. Langmuir 2001, 17, 2560-2563. 
(34) Ashcroft, N.W.; Mermin, N.D. Solid State Physics; Saunders College Publications: Philadelphia, 1976.

(35) Evans, S. D.; Ulman, A. Chem. Phys. Lett. 1990, 170, 462-466.

(36) Evans, S. D.; Urankar, E.; Ulman, A.; Ferris, N. J. Am. Chem. Soc. 1991, 113, 4121-4131.

(37) Landau, L. D.; Lifshitz, E. M.; Pitaevskii, L. P. Electrodynamics of Continuous Media, Second Edition; Butterworth Heinemann: New York, 1984.

(38) Cahn, J. W.; Hilliard, J. E. J. Chem. Phys. 1958, 28, 258-267.

(39) Berger, R.; Delamarche, E.; Lang, H. P.; Gerber, C.; Gimzewski, J. K.; Meyer, E.; Güntherodt, H.-J. Science 276, 1997, 2021- 2024.

(40) Chen, L.-Q.; Shen, J. Comp. Phys. Comm. 1998, 108, 147-158.

(41) Lu, W.; Suo, Z. Phys. Rev. B, in press. Preprints are available on www.princeton.edu/ suo (Pub 119 and Pub 122).

(42) Gao, Y. F.; Lu, W.; Suo, Z. submitted to Acta Mater. Preprint is available as above (Pub 124).

(43) Gao, Y. F.; Suo, Z. submitted to J. Mech. Phys. Solids. Preprint is available as above (Pub 128).

(44) Chou, S. Y.; Zhuang, L. J. Vac. Sci. Tech. B 1999, 17, 3197-3202.

(45) Schaffer, E.; Thurn-Albrecht, T.; Russell, T. P.; Steiner, U. Nature 2000, 403, 874-877.

(46) Suo, Z.; Liang, J. Appl. Phys. Lett. 2001, 78, 3971-3973. 


\section{Figure Captions}

Figure 1 When a metal is in contact with an alkanethiol solution, the alkanethiol molecules adsorb on the metal surface to form a monolayer. The structure of a $\mathrm{HS}\left(\mathrm{CH}_{2}\right)_{4} \mathrm{OH}$ molecule is illustrated.

Figure 2 The free energy of mixing for a monolayer composed of two molecular species, A and B. The pair has a large enthalpy of mixing, so that the free energy of mixing has two wells at $C_{\alpha}$ and $C_{\beta}$, corresponding to two phases. When the average concentration of the monolayer, $C_{0}$, is between the two wells, to reduce the free energy, the monolayer separates into the two phases.

Figure 3 The contact potential $U=\phi_{\beta}-\phi_{\alpha}$ causes an electrostatic field in the air, a positive charge on the metal surface under domain $\beta$, and a negative charge under domain $\alpha$. Represent the domain size by the period $\lambda$.

Figure 4 (a) The charge $Q$ accumulated under either domain increases linearly with the contact potential between the two domains, $U=\phi_{\beta}-\phi_{\alpha}$. The area of the triangle is the electrostatic energy stored in the space occupied by the air. The slope of the line is inverse of the capacitance of the system. (b) The $Q-U$ lines for two domain sizes, $\lambda_{1}<\lambda_{2}$. At a constant voltage, the smaller the domain size, the larger the charge, namely, $Q_{1}>Q_{2}$. (c) In a parallel-electrode capacitor, the electric interaction causes the attraction between two electrodes. To keep the two electrodes in place, one has to apply a pair of forces to pull the electrodes apart. 
Figure 5 The boundary conditions at the interfacial object between the air and the bulk of the metal.

Figure 6 (a) The concentration field initially fluctuates with small amplitude around the average concentration $C_{0}=0.5$, and evolves into a pattern of meandering stripes. (b) The concentration field initially fluctuates with small amplitude around the average concentration $C_{0}=0.4$, and evolves into a pattern of dots. 


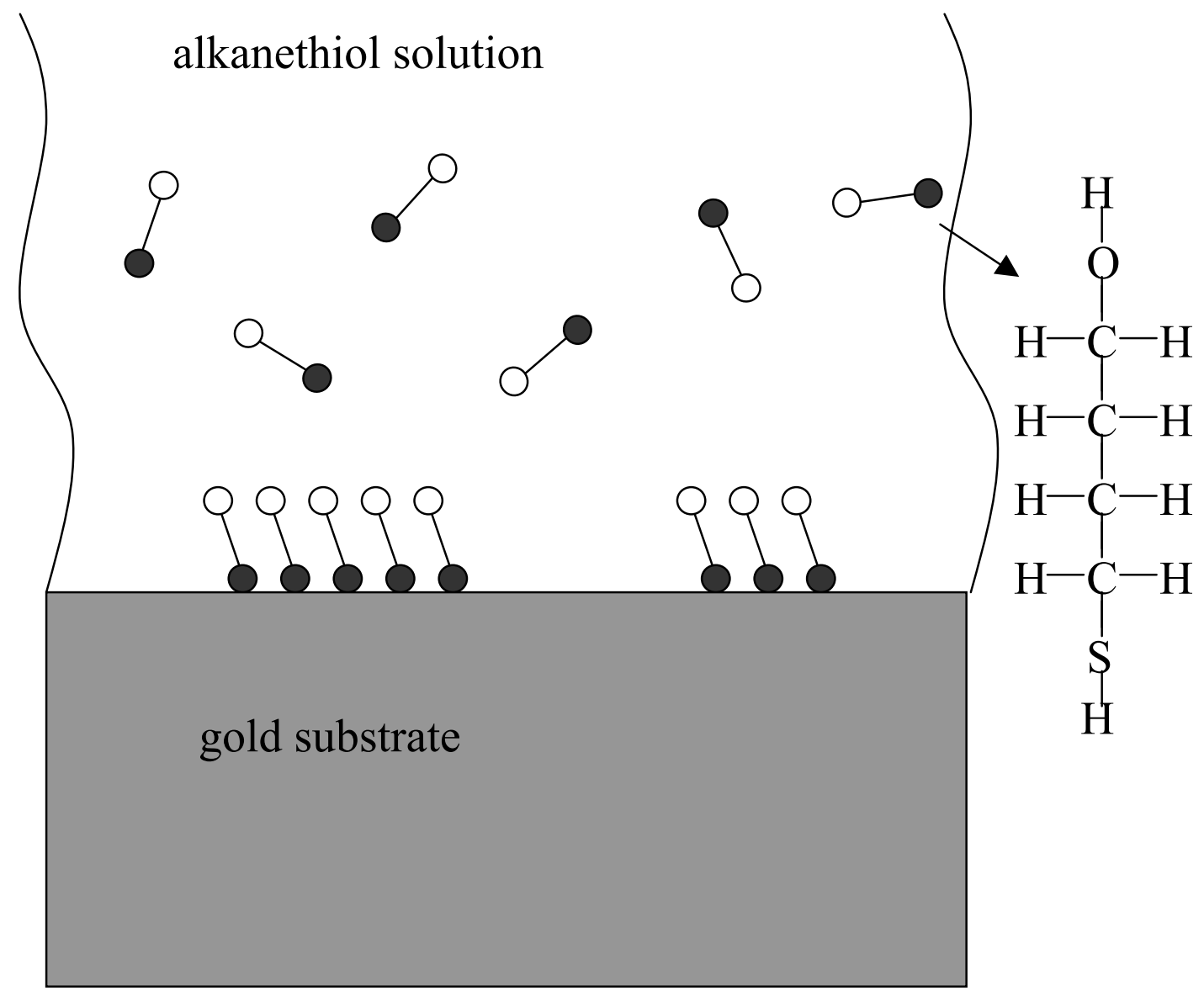

Fig. 1 


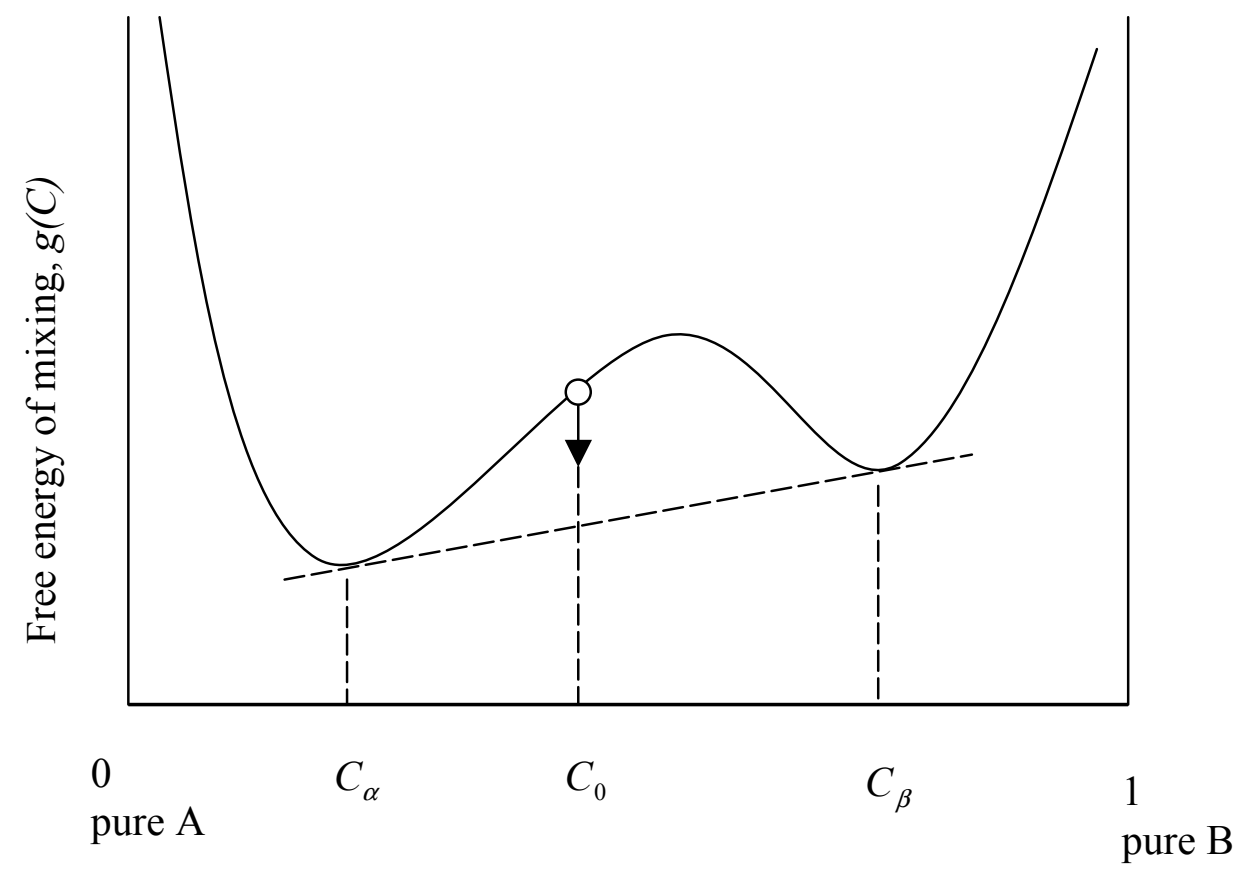

Fraction of molecule B in monolayer, $C$

Fig. 2 


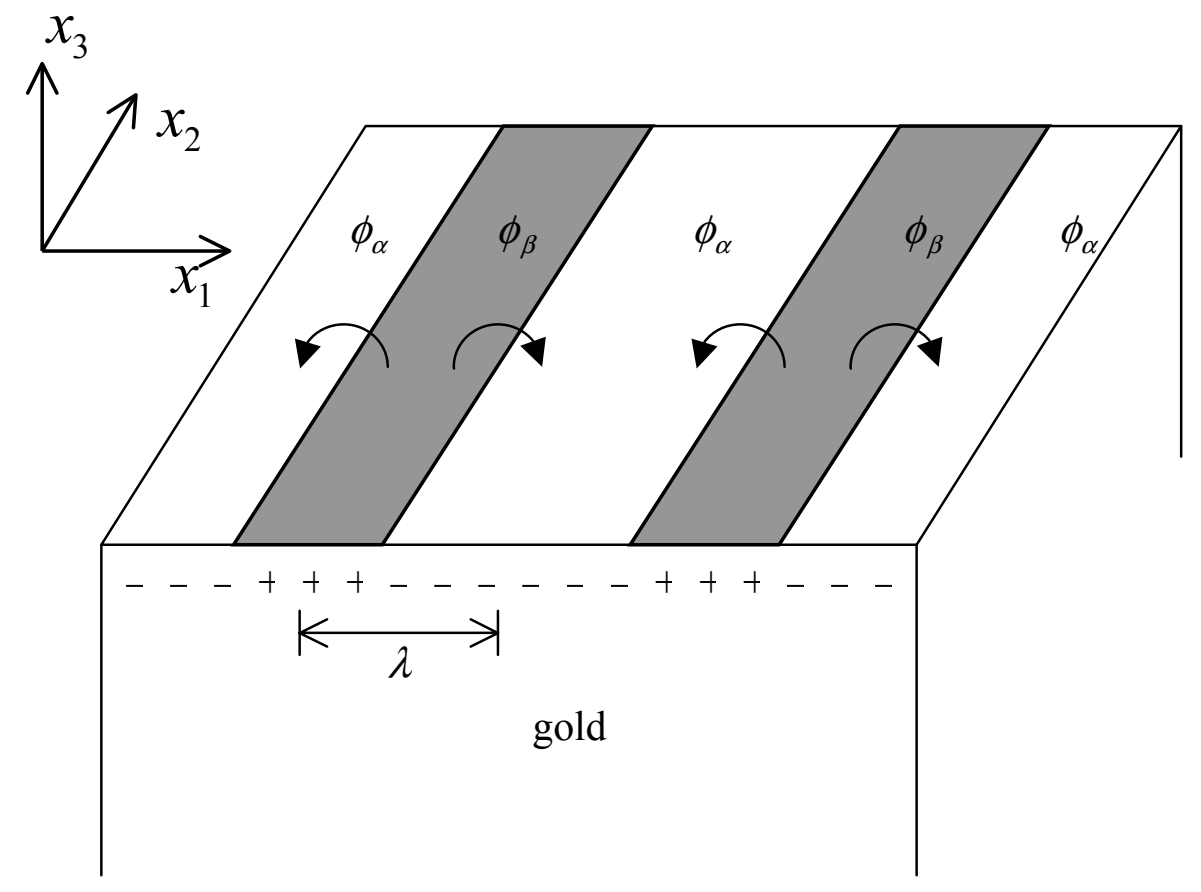

Fig. 3 
a)

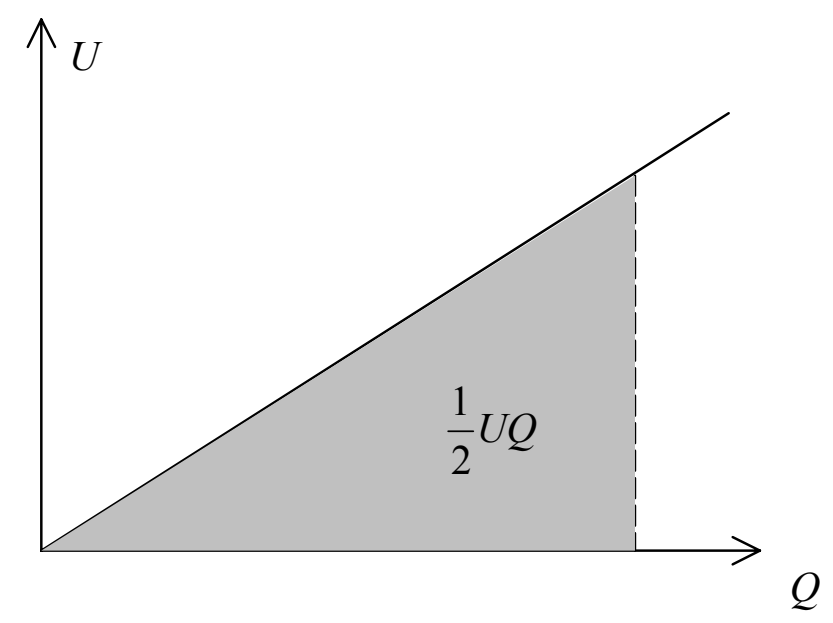

b)

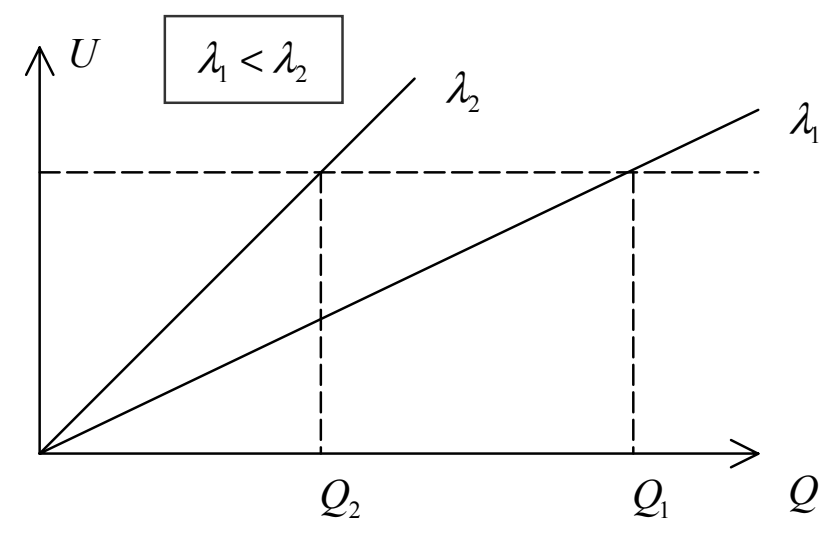

c)

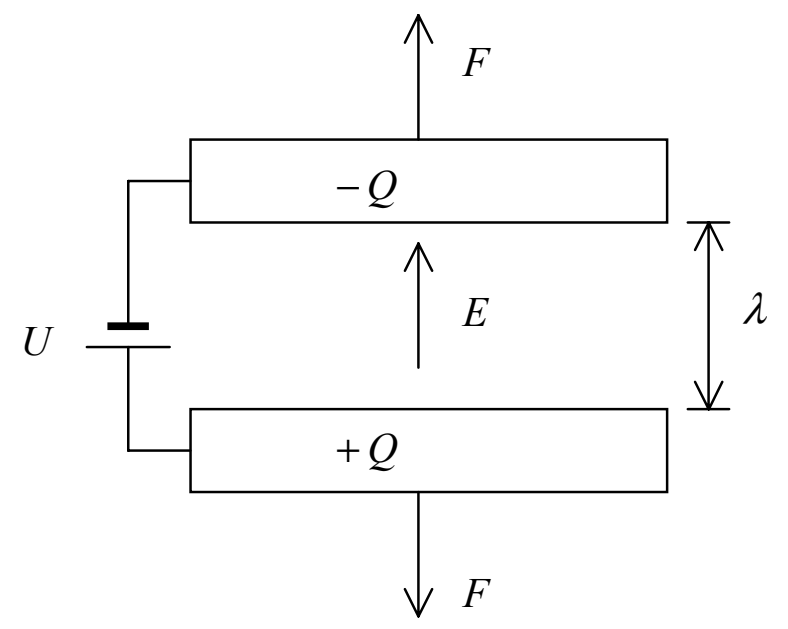

Fig. 4 


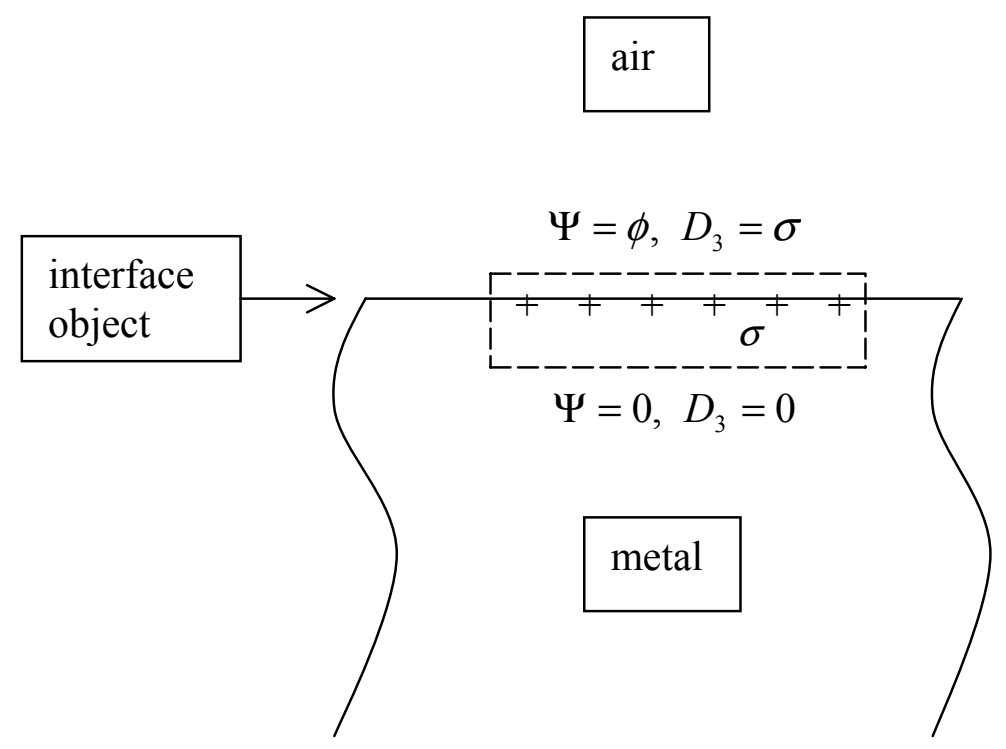

Fig. 5 
(a)

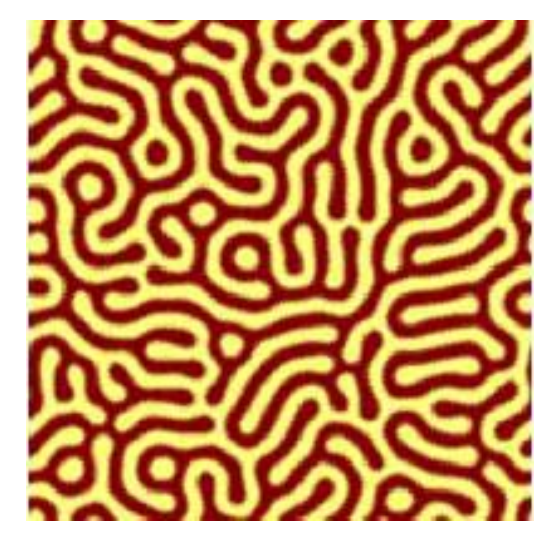

(b)

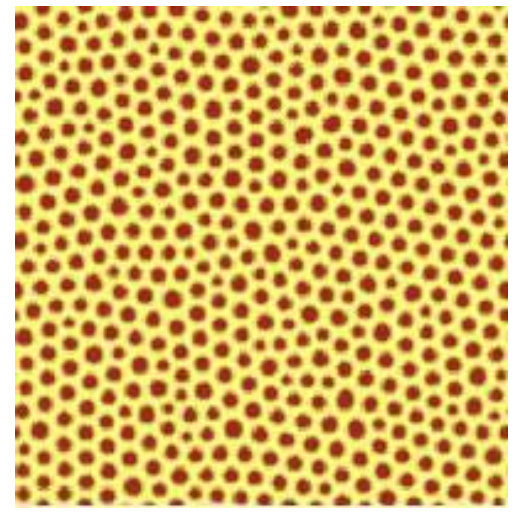

Fig. 6 